E3S Web of Conferences 1, 40001 (2013)

DOI: $10.1051 / \mathrm{e} 3$ sconf/20130140001

(C) Owned by the authors, published by EDP Sciences, 2013

\title{
Neurodevelopment of Infants in a Mercury Contaminated Crea in Zimbabwe
}

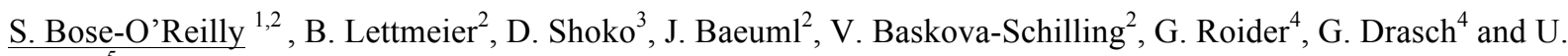 \\ Siebert ${ }^{5}$ \\ ${ }^{1}$ University Hospital Munich, Institute and Outpatient Clinic for Occupational, Social and Environmental Medicine, \\ WHO Collaborating Centre for Occupational Health, Munich, Germany; Stephan.Boeseoreilly@med.uni-muenchen.de \\ ${ }^{2}$ Institute of Public Health, Medical Decision Making and Health Technology Assessment, Department of Public Health \\ and Health Technology Assessment, University for Health Sciences, Medical Informatics and Technology (UMIT), Hall \\ i.T., Austria; beate.lettmeier@umit.at, jennifer.baeuml@umit.at, torybaskova@yahoo.com , uwe.siebert@umit.at \\ ${ }^{3}$ University of Zimbabwe, Harare, Zimbabwe; dennis.shoko@gmail.com \\ ${ }^{4}$ Institute of Forensic Medicine, Ludwig-Maximilians-University (LMU), \\ ${ }^{5}$ Institute of Forensic Medicine, Ludwig-Maximilians-University, Nussbaumstr. 26, D-80336 Munich, Germany \\ Gabriele.Roider@med.uni-muenchen.de,drasch@allacher-apotheke.de
}

\begin{abstract}
In Kadoma in Zimbabwe small-scale gold mining is widespread. Mothers and their infants are exposed in the mining communities to the toxic inorganic mercury vapors from processing the ore using elemental mercury. Methyl-mercury is a well known neurotoxin and endangers the regular development of children. This study examined the influence of exposure to elemental mercury for breast fed infants in Kadoma. 121 mother-child pairs were selected, 40 from a non-exposed control area, 81 from the exposed area in Kadoma. Urine, hair and breast milk was analyzed for total mercury. Mainly urine and breast milk levels were significantly higher for the exposed infants. The infants were clinically examined, and their developmental status assessed. In relation to the exposure markers in urine and breast milk several developmental parameters showed statistically significant negative results, such as the capability of infants to sit, crawl or stand at a certain age. These analyzes indicate that a higher body burden with elemental mercury decreases the speed of a regular development of infants.
\end{abstract}

Keywords: Neurodevelopment, mercury vapor, breast milk, small scale gold mining, Zimbabwe

\section{Introduction}

In gold mining areas all over the word, small miners extract the gold from ore using mercury. In Kadoma in Zimbabwe small-scale gold mining is very common. Mercury can cause acute and chronic intoxication. Of major concern is the developing brain before and after birth. An environmental epidemiological study was conducted by LMU and UMIT. One aim of the study was to investigate whether the exposure to mercury of breast fed children with inorganic mercury in small scale gold mining areas affects the neurodevelopment of these infants.

\section{Methods}

In 2006, 121 mother-child pairs from the exposed Kadoma area and from a non-exposed control area in
Chikwaka were randomly selected to participate voluntarily in the study. Only mother-infant pairs were considered, with breast fed children between 3 and 9 months of age. The population was divided into three subgroups: A non exposed control group Chikwaka, were no gold mining activities occur (40 participants); a medium exposed group with mothers living in Kadoma but not working with mercury (53 participants); a high exposed group with mothers living in Kadoma and working with mercury, e.g. panning the ore or smelting amalgam (28 participants).

A questionnaire was used to assess the exposure situation, possible confounders, the general health and nutrition status and specific anamnestic questions related to mercury. All children received a full examination, similar to regular health check-ups for infants, e.g. heart, lung, abdomen, skin, genitals, skeletal system, ears, eyes and mouth. All children were neurologically examined, 
including muscular tonus and reflexes. The following neuropsychological tests were carried out with the children: Munich functional developmental test to determine the developmental stage; WHO assessment of motor milestone to determine the developmental stage.

Breast milk samples, hair and urine samples from mothers and children were selected and analyzed for total mercury in the laboratory in Munich.

As expected, the concentration of mercury in hair, urine and breast milk was distributed with a tail to the right. Chi-square (Pearson) was used to test for any difference between the different exposure groups for clinical ordinal data. The statistical analysis of metric data was performed with T-Test, ANOVA, Kruskal Wallis and Mann Whitney test. Logistic regression was used to find predictors between clinical parameters and toxicological thresholds limits. The achievement of milestones was tested with Levene's test to assess the parity of variance in different samples.

\section{Results}

Analyzing the urine with atomic absorption spectrometry gave the total amount of mercury. For mothers the median level was $0.10[\mu \mathrm{g} / 1]$ for the control group, for the medium exposed group the median was $4.00[\mu \mathrm{g} / 1]$, and for the high exposed group $8.56[\mu \mathrm{g} / \mathrm{l}]$; maximum level $441[\mu \mathrm{g} / 1]$. For children the median level was $0.36[\mu \mathrm{g} / \mathrm{l}]$ for the control group, for the medium exposed group the median was $1.40[\mu \mathrm{g} / 1]$, and for the high exposed group $4.42[\mu \mathrm{g} / 1]$; maximum level $86.9[\mu \mathrm{g} / 1]$. The results for total mercury in urine in relation to the excretion of creatinine were for mothers as follows. The median level was $0.03[\mu \mathrm{g} / \mathrm{g}$ crea] for the control group, for the medium exposed group the median was $2.54[\mu \mathrm{g} / \mathrm{g}$ crea], and for the high exposed group $6.85[\mu \mathrm{g} / \mathrm{g}$ crea]; maximum level $286[\mu \mathrm{g} / \mathrm{g}$ crea]. For children the median level was $1.43[\mu \mathrm{g} / \mathrm{g}$ crea] for the control group, for the medium exposed group the median was $9.15[\mu \mathrm{g} / \mathrm{g}$ crea], and for the high exposed group 28.9 [ $\mu \mathrm{g} / \mathrm{g}$ crea]; maximum level 258 [ $\mu \mathrm{g} / \mathrm{g}$ crea].

The median level for total mercury in hair for mothers was in the control group $0.08[\mu \mathrm{g} / \mathrm{g}]$, in the medium exposed group $1.10[\mu \mathrm{g} / \mathrm{g}]$, in the high exposed group $1.47[\mu \mathrm{g} / \mathrm{g}]$, maximum level was $27.8[\mu \mathrm{g} / \mathrm{g}]$. The median level for total mercury in hair for children was in the control group $0.14[\mu \mathrm{g} / \mathrm{g}]$, in the medium exposed group $0.94[\mu \mathrm{g} / \mathrm{g}]$, in the high exposed group $1.24[\mu \mathrm{g} / \mathrm{g}]$, maximum level was $9.70[\mu \mathrm{g} / \mathrm{g}]$.

The median of the breast milk samples was $0.50 \mathrm{Hg}$ $[\mu \mathrm{g} / \mathrm{l}]$ in the control group, $1.10 \mathrm{Hg}[\mu \mathrm{g} / \mathrm{l}]$ in the medium exposed group and $1.30 \mathrm{Hg}[\mu \mathrm{g} / \mathrm{l}]$ in the high exposed group, the maximum level was $24.80 \mathrm{Hg}$ [ $\mu \mathrm{g} / \mathrm{l}]$. The daily uptake of mercury from breast milk of the children was calculated. The reference dose of $0.3^{\circ}[\mu \mathrm{g} \mathrm{Hg} / \mathrm{kg} \mathrm{BW} / \mathrm{d}]$ was used to compare the uptake of the infants in this study. No infant from the control area, 9 infants or $17 \%$ in the medium exposed group and 6 infants or $21 \%$ in the high exposed group have an daily uptake of mercury via breast-milk above the RfD. The difference versus the control group is significant $(\mathrm{p}=0.012)$.

Comparing the control group with the exposed participants there are significantly more problems during pregnancy $(0 \%$ versus $11 \%)$ and during delivery $(0 \%$ versus $12 \%$ ). The questionnaire about the general health status of mothers and children showed only one difference, a higher rate of malaria for the two exposed groups. Furthermore a general check up of the children was performed. The two exposed group had significantly more genital abnormalities $(0 \%$ non exposed, $6 \%$ medium exposed and $18 \%$ high exposed group).

The assessment of the developmental milestones of the children did not reveal any difference if tested only versus the subgroup with a Chi-square (Pearson) test. But achieving a developmental milestone, such as sitting, crawling, standing or walking depends strongly on the age of the child. The children's development was assessed, using a WHO assessment scheme. As to be expected the achievement of the developmental milestones such as sitting, crawling or walking did depend on the age of the child. The hypothesis was that the higher burdened group would have a delayed development, which could not be shown using the three subgroups. Another analysis, using the reference value of mercury in children's urine $0.70[\mu \mathrm{g} / 1]$ as decision point shows a difference between the group below the reference value versus the group above the reference group. Statistical analysis with Levene test indicates that the difference is significant for the milestone crawling. Using breast milk's reference dose $0.30[\mu \mathrm{g} / \mathrm{kh} \mathrm{bw} / \mathrm{d}]$ as decision point shows a difference for sitting and walking, which is significant using Levene test. These analyzes indicate that a higher body burden with mercury decreases the speed of a regular development of infants.

\section{Discussion}

The number of examined mother child pairs was not large, and the regular achievement of the developmental milestones does naturally differ between children. Nevertheless this study shows, that the developmental milestones of exposed children were delayed compared to lesser exposed children. Mercury is known as a neurotoxic substance. But is a new result, that the children in gold mining areas, exposed mainly to inorganic mercury do show neurodevelopmental effects, similar to the known exposure with methylmercury via fish. 